\title{
Clustering of Dependent Components: A New Paradigm for fMRI Signal Detection
}

\author{
Anke Meyer-Bäse \\ Department of Electrical and Computer Engineering, Florida State University, Tallahassee, FL 32310-6046, USA
}

Email:amb@eng.fsu.edu

Monica K. Hurdal

Department of Mathematics, Florida State University, Tallahassee, FL 32306-4510, USA

Email:mhurdal@math.fsu.edu

\author{
Oliver Lange \\ Department of Electrical and Computer Engineering, Florida State University, Tallahassee, FL 32310-6046, USA \\ Email: oliver@lange.org
}

\author{
Helge Ritter \\ Neuroinformatics Group, Faculty of Technology, University of Bielefeld, 33501 Bielefeld, Germany \\ Email:helge@techfak.uni-bielefeld.de
}

Received 1 February 2004

\begin{abstract}
Exploratory data-driven methods such as unsupervised clustering and independent component analysis (ICA) are considered to be hypothesis-generating procedures and are complementary to the hypothesis-led statistical inferential methods in functional magnetic resonance imaging (fMRI). Recently, a new paradigm in ICA emerged, that of finding "clusters" of dependent components. This intriguing idea found its implementation into two new ICA algorithms: tree-dependent and topographic ICA. For fMRI, this represents the unifying paradigm of combining two powerful exploratory data analysis methods, ICA and unsupervised clustering techniques. For the fMRI data, a comparative quantitative evaluation between the two methods, tree-dependent and topographic ICA, was performed. The comparative results were evaluated by (1) task-related activation maps, (2) associated time courses, and (3) ROC study. The most important findings in this paper are that (1) both tree-dependent and topographic ICA are able to identify signal components with high correlation to the fMRI stimulus, and that (2) topographic ICA outperforms all other ICA methods including tree-dependent ICA for 8 and 9 ICs. However for 16 ICs, topographic ICA is outperformed by treedependent ICA (KGV) using as an approximation of the mutual information the kernel generalized variance. The applicability of the new algorithm is demonstrated on experimental data.
\end{abstract}

Keywords and phrases: dependent component analysis, topographic ICA, tree-dependent ICA, fMRI.

\section{INTRODUCTION}

Functional magnetic resonance imaging with high temporal and spatial resolution represents a powerful technique for visualizing rapid and fine activation patterns of the human brain $[1,2,3,4,5]$. As is known from both theoretical estimations and experimental results $[4,6,7]$, an activated signal variation appears very low on a clinical scanner. This motivates the application of analysis methods to determine the response waveforms and associated activated regions. Generally, these techniques can be divided into two groups: model-based techniques require prior knowledge about activation patterns, whereas model-free techniques do not. However, model-based analysis methods impose some limitations on data analysis under complicated experimental conditions. Therefore, analysis methods that do not rely on any assumed model of functional response are considered more powerful and relevant. We distinguish two groups of model-free methods: transformation-based and clusteringbased methods. There are two kinds of model-free methods. The first kind, principal component analysis (PCA) $[8,9]$ or independent component analysis (ICA) $[10,11,12,13]$, transforms original data into high-dimensional vector space to separate functional response and various noise sources from each other. The second kind, fuzzy clustering analysis $[14,15,16,17]$ or self-organizing maps $[17,18,19]$, 
attempts to classify time signals of the brain into several patterns according to temporal similarity among these signals.

Among the data-driven techniques, ICA has been shown to provide a powerful method for the exploratory analysis of fMRI data $[11,13]$. ICA is an information-theoretic approach which enables to recover underlying signals, or independent components (ICs) from linear data mixtures. Therefore, it is an excellent method to be applied for the spatial localization and temporal characterization of sources of BOLD activation. ICA can be applied to fMRI both temporally and spatially. Spatial ICA has dominated so far in fMRI applications because the spatial dimension is much larger than the temporal dimension in fMRI. However, recent literature results have suggested that temporal and spatial ICA yield similar results for experiments where two predictable task-related components are present.

A new methodology has attracted a lot of attention in the ICA community during the last two years: the idea of finding "clusters" of independent components. Two leading papers implemented this new paradigm in a striking way. Clusters are defined as connected components of a graphical model (lattice in [20] and tree structured in [21]). Both models attempt a decomposition of the source variables such that they are dependent within a cluster and independent between the clusters. This idea emerged from multidimensional ICA, where the sources are not assumed to be all mutually independent [22]. Instead, it is assumed that they can be grouped in $n$-tuples, such that within these tuples they are dependent on each other, but are independent outside.

The two paradigms differ in terms of topology and the knowledge of number and sizes of components.

In [20], the components are arranged on a two-dimensional grid or lattice as is typical in topographic models. The goal is to define a statistical model where the topographic proximity reflects the statistical dependencies between components. The components (simple cells) are placed on the grid such that any two cells that are close to each other model dependent components whereas cells that are far from each other model independent components. The measure of dependency is based on the correlation of energies. Energy in this context means the squaring operation. Nonlinear correlations are of importance since they cannot be easily set to zero by standard whitening procedures. Translated to our model, this means that energies are strongly positively correlated for neighboring components. The topology of the model is fixed. This model also requires that the number and sizes of the components have to be fixed in advance. Learning is based on the maximization of the likelihood.

A totally different concept is employed in [21]. Here, the topology of the dependency structure is not fixed in advance. However, it is assumed that it has the structure of a tree. The goal of the learning is to identify a minimal spanning tree connecting the given sources in such a manner that no other tree expresses the dependency structure of the given distribution better. It is interesting to point out that in traditional ICA the graphical model has no edges meaning that the random variables are mutually independent.
We have seen that both clustering methods as well as ICA techniques have their particular strengths in fMRI signal detection. Therefore, it is natural to look for a unifying technique that combines those two processing mechanisms and applies this combination to fMRI. The topographic and the tree-dependent ICA, as previously described, have the computational advantages associated with both techniques.

In this paper, we perform a detailed comparative study for fMRI among the tree-dependent and topographic ICA with standard ICA techniques. In a systematic manner, we will compare and evaluate the results obtained based on each technique and present the benefits associated with each paradigm.

\section{EXPLORATORY DATA ANALYSIS METHODS}

Functional organization of the brain is based on two complementary principles, localization and connectionism. Localization means that each visual function is performed mainly by a small set of cortical neurons. Connectionism, on the other hand, expresses that the brain regions involved in a certain visual cortical function are widely distributed, and thus the brain activity necessary to perform a given task may be the functional integration of activity in distinct brain systems. It is important to stress that in neurobiology the term "connectionism" is used in a different sense than that used in the neural network terminology.

The following sections are dedicated to presenting the algorithms and evaluate the discriminatory power of the two main groups of exploratory data analysis methods.

\subsection{The basic ICA algorithms}

According to the principle of functional organization of the brain, it was suggested for the first time in [11] that the multifocal brain areas activated by the performance of a visual task should be unrelated to the brain areas whose signals are affected by artifacts of physiological nature, head movements, or scanner noise related to fMRI experiments. Every single above-mentioned signal can be described by one or more spatially independent components, each associated with a single time course of a voxel and a component map. It is assumed that the component maps, each described by a spatial distribution of fixed values, are representing overlapping, multifocal brain areas of statistically dependent fMRI signals. This aspect is visualized in Figure 1. In addition, it is considered that the distributions of the component maps are spatially independent and, in this sense, uniquely specified. Mathematically, this means that if $p_{k}\left(C_{k}\right)$ specifies the probability distribution of the voxel values $C_{k}$ in the $k$ th component map, then the joint probability distribution of all $n$ components yields

$$
p\left(C_{1}, \ldots, C_{m}\right)=\prod_{k=1}^{n} p_{k}\left(C_{k}\right)
$$

where each of the component maps $C_{k}$ is a vector $\left(C_{k i}, i=\right.$ $1,2, \ldots, M)$, where $M$ gives the number of voxels. Independency is a stronger condition than uncorrelatedness. It was 


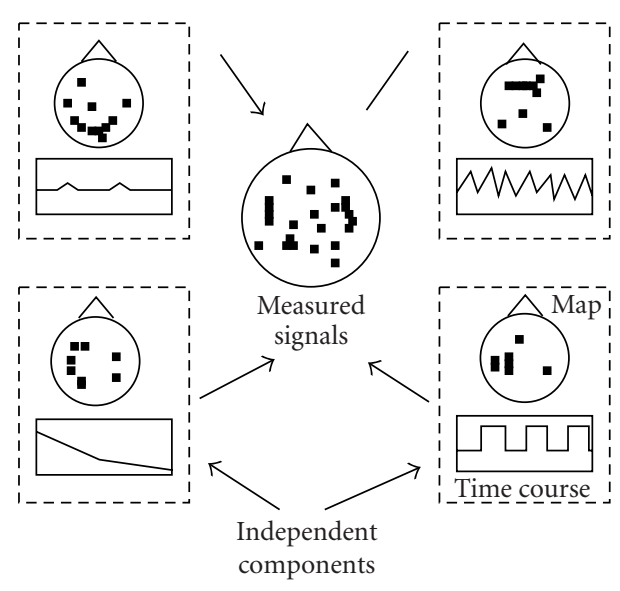

(a)

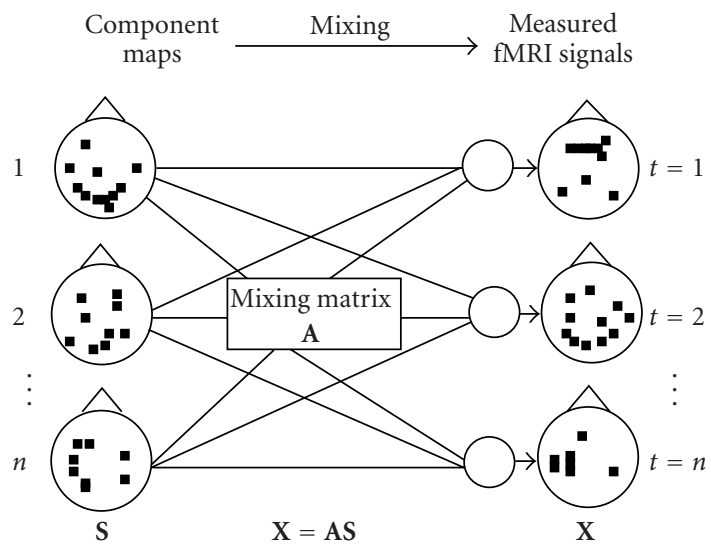

(b)

FIgURE 1: Visualization of ICA applied to fMRI data. (a) Scheme of fMRI data decomposed into independent components, and (b) fMRI data as a mixture of independent components where the mixing matrix A specifies the relative contribution of each component at each time point [11].

shown in [11] that these maps are independent if the active voxels in the maps are sparse and mostly nonoverlapping. Additionally it is assumed that the observed fMRI signals are the superposition of the individual component processes at each voxel. Based on these assumptions, ICA can be applied to fMRI time series to spatially localize and temporally characterize the sources of BOLD activation.

Different methods for performing ICA decompositions have been proposed which employ different objective functions together with different criteria of optimization of these functions, and it is assumed that they can produce different results.

\subsection{Models of spatial ICA in FMRI}

In the following we will assume that $\mathrm{X}$ is a $T \times M$ matrix of observed voxel time courses (fMRI signal data matrix), $\mathrm{C}$ is the $N \times M$ random matrix of component map values, and A is a $T \times N$ mixing matrix containing in its columns the associated time courses of the $N$ components. Furthermore, $T$ corresponds to the number of scans, and $M$ is the number of voxels included in the analysis.

The spatial ICA (sICA) problem is given by the following linear combination model for the data:

$$
\mathrm{X}=\mathrm{AC}
$$

where no assumptions are made about the mixing matrix $\mathbf{A}$ and the rows $\mathbf{C}_{i}$ being mutually statistically independent.

Then the ICA decomposition of $\mathbf{X}$ can be defined as an invertible transformation:

$$
\mathbf{C}=\mathbf{W X},
$$

where $\mathbf{W}$ is an unmixing matrix providing a linear decomposition of data. $\mathbf{A}$ is the pseudoinverse of $\mathbf{W}$.
The employed ICA algorithms are the TDSEP, JADE, and the FastICA approach based on the minimization of mutual information but using the negentropy as a measure of nonGaussianity [23], and topographic ICA which combines topographic mapping with ICA [20].

\subsection{Tree-dependent component analysis model}

The paradigm of TCA is derived from the theory of treestructured graphical models. In [24] a strategy was shown to approximate optimally an $n$-dimensional discrete probability distribution by a product of second-order distributions, or the distribution of the first-order tree dependence. A tree is an undirected graph with at most a single edge between two nodes. This tree concept can be easily interpreted with respect to ICA. A graph with no edges means that the random variables are mutually independent and this pertains to ICA. On the other hand, if no assumptions are made about independence, then the corresponding family of probability distributions represents the set of all distributions.

A probability distribution can be approximated in several ways. Here, we look into approximations based on a product of $n-1$ second-order component distributions. In [24] a strategy of the best approximation of an $n$ th-order distribution was developed by a product of $n-1$ second-order component distributions:

$$
P_{i}(\mathbf{x})=\prod_{i=1}^{n} P\left(x_{m_{i}} \mid x_{m_{j}(i)}\right), \quad 0 \leq j(i)<i,
$$

where $P(\mathbf{x})$ is a joint probability distribution of $n$ discrete variables with $\mathbf{x}=x_{1}, \ldots, x_{n}$ being a vector, $\left(m_{1}, \ldots, m_{n}\right)$ is an unknown permutation of integers $1,2, \ldots, n$, and $P\left(x_{i} \mid x_{0}\right)$ is by definition equal to $P\left(x_{i}\right)$. The above introduced probability distribution is named a probability distribution of firstorder tree dependence. 
To determine the goodness of an approximation, it is necessary to define a closeness as

$$
I\left(P, P_{a}\right)=\sum_{\mathbf{x}} P(\mathbf{x}) \log \frac{P(\mathbf{x})}{P_{a}(\mathbf{x})},
$$

where $P(\mathbf{x})$ and $P_{a}(\mathbf{x})$ are two probability distributions of the $n$ random variables $\mathbf{x}$. The quantity $I\left(P, P_{a}\right)$ has the property $I\left(P, P_{a}\right) \geq 0$.

Translated to random variables, the above definition is named mutual information and is always nonnegative:

$$
I\left(x_{i}, x_{j}\right)=\sum_{x_{i}, x_{j}} P\left(x_{i}, x_{j}\right) \log \left(\frac{P\left(x_{i}, x_{j}\right)}{P\left(x_{i}\right) P\left(x_{j}\right)}\right) .
$$

In the following, we will state the solution to the approximation of the probability distribution. We are searching for a distribution of tree dependence $P_{\tau}\left(x_{1}, \ldots, x_{n}\right)$ such that $I\left(P, P_{\tau}\right) \leq I\left(P, P_{t}\right)$ for all $t \in T_{n}$ where $T_{n}$ represents the set of all possible first-order dependence trees. Thus, the solution $\tau$ is defined as the optimal first-order dependence tree.

In parlance of graph theory, every branch of the dependence tree is assigned a branch weight $I\left(x_{i}, x_{j(i)}\right)$. Thus being given a dependence tree $t$, the sum of all branch weights becomes a useful quantity.

In [24] it was shown that a maximum-weight dependence tree is a dependence tree $t$ such that, for all $t^{\prime}$ in $T_{n}$,

$$
\sum_{i=1}^{n} I\left(x_{i}, x_{j}(i)\right) \geq \sum_{i=1}^{n} I\left(x_{i}, x_{j^{\prime}(i)}\right)
$$

In other words, a probability distribution of tree dependence $P_{t}(\mathbf{x})$ is an optimum approximation to $P(\mathbf{x})$ if and only if its dependence tree $t$ has maximum weight or minimizing the closeness measure $I\left(P, P_{t}\right)$ is equivalent to maximizing the total branch weight.

The idea of approximating discrete probability distributions with dependence trees described before and adapted from [24] can be easily translated to ICA [21].

In classic ICA, we want to minimize the mutual information of the estimated components $\mathbf{s}=\mathbf{W} \mathbf{x}$. Thus, the result derived in [24] can be easily extended and becomes the treedependent ICA.

The objective function for TCA is given by $J(\mathbf{x}, \mathbf{W}, t)$ and includes the demixing matrix $\mathbf{W}$. Thus, the mutual information for TCA becomes

$$
J(\mathbf{x}, \mathbf{W}, t)=I^{t}(\mathbf{s})=I\left(s_{1}, \ldots, s_{m}\right)-\sum_{(u, v) \in t} I\left(s_{u}, s_{v}\right)
$$

where $\mathbf{s}$ factorizes in a tree $t$.

In TCA as in ICA, the density $p(\mathbf{x})$ is not known and the estimation criteria have to be substituted by empirical contrast functions. As described in [21], we will employ three types of contrast functions: (i) approximation of the entropiesbeing part of (8) via kernel density estimation
(KDE), (ii) approximation of the mutual information based on kernel generalized variance (KGV), and (iii) approximation based on cumulants using Gram-Charlier expansions (CUM).

\subsection{Topographical independent component analysis}

The topographic independent component analysis [20] represents a unifying model which combines topographic mapping with ICA.

Achieved by a slight modification of the ICA model, it can at the same time be used to define a topographic order between the components and thus has the usual computational advantages associated with topographic maps.

The paradigm of topographic ICA has its roots in [25] where a combination of invariant feature subspaces [26] and independent subspaces [22] is proposed. In the following, we will describe these two parts, which substantially reflect the concept of topographic ICA [27].

\subsubsection{Invariant feature subspaces}

The principle of invariant feature subspaces was developed by Kohonen [26] with the intention of representing features with some invariances. This principle states that an invariant feature is given by a linear subspace in a feature space. The value of the invariant feature is given by the squared norm of the projection of the given data point on that subspace.

A feature subspace can be described by a set of orthogonal basis vectors $\mathbf{w}_{j}, j=1, \ldots, n$, where $n$ is the dimension of the subspace. Then the value $G(\mathbf{x})$ of the feature $G$ with the input vector $\mathbf{x}$ is given by

$$
G(\mathbf{x})=\sum_{j=1}^{n}\left\langle\mathbf{w}_{j}, \mathbf{x}\right\rangle^{2}
$$

In other words, this describes the distance between the input vector $\mathbf{x}$ and a general linear combination of the basis vectors $\mathbf{w}_{j}$ of the feature subspace [26].

\subsubsection{Independent subspaces}

Traditional ICA works under the assumption that the observed signals $x_{i}(t)(i=1, \ldots, n)$ are generated by a linear weighting of a set of $n$ statistically independent random sources $s_{j}(t)$ with time-independent coefficients $a_{i j}$. In a matrix form, this can be expressed as

$$
\mathbf{x}(t)=\mathbf{A s}(t)
$$

where $\mathbf{x}(t)=\left[x_{1}(t), \ldots, x_{n}(t)\right]^{T}, \mathbf{s}(t)=\left[s_{1}(t), \ldots, s_{n}(t)\right]$, and $\mathbf{A}=\left[a_{i j}\right]$.

In multidimensional ICA [22], the sources $s_{i}$ are not assumed to be all mutually independent. Instead, it is assumed that they can be grouped in $n$-tuples, such that within these tuples they are dependent on each other, but are independent outside. This newly introduced assumption was observed in several image processing applications. Each $n$-tuple of sources $s_{i}$ corresponds to $n$ basis vectors given by the rows 
of matrix A. A subspace spanned by a set of $n$ such basis vectors is defined as an independent subspace. In [22] two simplifying assumptions are made: (1) although $s_{i}$ are not at all independent, they are chosen to be uncorrelated and of unit variance, and (2) the data are preprocessed by whitening (sphering) them. This means the $\mathbf{w}_{j}$ are orthonormal.

Let $J$ be the number of independent feature subspaces and $S_{j}, j=1, \ldots, J$, the set of indices that belong to the subspace of index $j$. Assume that we have $T$ given observations $\mathbf{x}(t), t=1, \ldots, T$. Then the likelihood $L$ of the data based on the model is given by

$$
\begin{aligned}
L\left(\mathbf{w}_{i},\right. & i=1, \ldots, n) \\
= & \prod_{t=1}^{T}\left[|\operatorname{det} \mathbf{W}| \prod_{j=1}^{J} p_{j}\left(\left\langle\mathbf{w}_{i}, \mathbf{x}(t)\right\rangle, i \in S_{j}\right)\right]
\end{aligned}
$$

with $p_{j}(\cdot)$ being the probability density inside the $j$ th $n$-tuple of $s_{i}$. The expression $|\operatorname{det} \mathbf{W}|$ is due to the linear transformation of the pdf. As always with ICA, $p_{j}(\cdot)$ need not be known in advance.

\subsubsection{Fusion of invariant feature and independent subspaces}

In [25] it is shown that a fusion between the concepts of invariant and independent subspaces can be achieved by considering probability distributions for the $n$-tuples of $s_{i}$ being spherically symmetric, that is, depending on the norm. In other words, the pdf $p_{j}(\cdot)$ has to be expressed as a function of the sum of the squares of the $s_{i}, i \in S_{j}$, only. Additionally, it is assumed that the pdfs are equal for all subspaces.

The log likelihood of this new data model is given by

$$
\begin{aligned}
\log L\left(\mathbf{w}_{i}, i=1, \ldots, n\right) \\
\quad=\sum_{t=1}^{T} \sum_{j=1}^{J} \log p\left(\sum_{i \in S_{j}}\left\langle\mathbf{w}_{i}, \mathbf{x}(t)\right\rangle^{2}\right)+T \log |\operatorname{det} \mathbf{W}| .
\end{aligned}
$$

$p\left(\sum_{i \in S_{j}} s_{i}^{2}\right)=p_{j}\left(s_{i}, i \in S_{j}\right)$ gives the pdf inside the $j$ th $n$ tuple of $s_{i}$. Based on the prewhitening, we have $\log |\operatorname{det} \mathbf{W}|=$ 0 .

For computational simplification, set

$$
G\left(\sum_{i \in S_{j}} s_{i}^{2}\right)=\log p\left(\sum_{i \in S_{j}}\left\langle\mathbf{w}_{i}, \mathbf{x}(t)\right\rangle^{2}\right)
$$

Since it is known that the projection of visual data on any subspace has a super-Gaussian distribution, the pdf has to be chosen to be sparse. Thus, we will choose $G(u)=\alpha \sqrt{u}+\beta$ yielding a multidimensional version of an exponential distribution. $\alpha$ and $\beta$ are constants and enforce that $s_{i}$ is of unit variance.

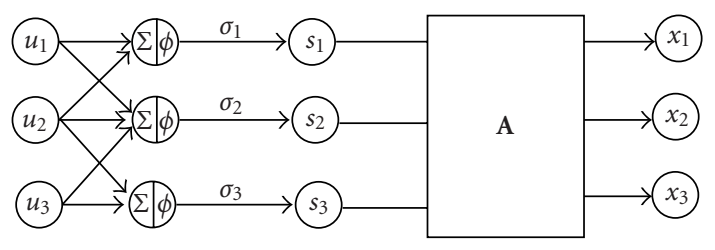

Figure 2: Topographic ICA model [20]. The variance-generating variables $u_{i}$ are randomly generated and mixed linearly within their topographic neighborhoods. This forms the input to nonlinearity $\phi$, thus giving the local variance $\sigma_{i}$. Components $s_{i}$ are generated with variances $\sigma_{i}$. The observed variables $x_{i}$ are obtained as with standard ICA from the linear mixture of the components $\mathrm{s}_{i}$.

\subsubsection{The topographic ICA architecture}

Based on the concepts introduced in the preliminary subsections, this section describes the topographic ICA.

To introduce a topographic representation in the ICA model, it is necessary to relax the assumption of independence among neighboring components $s_{i}$. This makes it necessary to adopt an idea from self-organized neural networks, that of a lattice. It was shown in [20] that a representation which models topographic correlation of energies is an adequate approach for introducing dependencies between neighboring components.

In other words, the variances corresponding to neighboring components are positively correlated while the other variances are, in a broad sense, independent. The architecture of this new approach is shown in Figure 2.

This idea leads to the following representation of the source signals:

$$
s_{i}=\sigma_{i} z_{i}
$$

where $z_{i}$ is a random variable having the same distribution as $s_{i}$, and the variance $\sigma_{i}$ is fixed to unity.

The variance $\sigma_{i}$ is further modeled by a nonlinearity:

$$
\sigma_{i}=\phi\left(\sum_{k=1}^{n} h(i, k) u_{k}\right)
$$

where $u_{i}$ are the higher-order independent components used to generate the variances, while $\phi$ describes some nonlinearity. The neighborhood function $h(i, k)$ can either be a twodimensional grid or have a ring-like structure. Further $u_{i}$ and $z_{i}$ are all mutually independent.

The learning rule is based on the maximization of the likelihood. First, it is assumed that the data are preprocessed by whitening and that the estimates of the components are uncorrelated. The log likelihood is given by

$$
\begin{aligned}
\log L & \left(\mathbf{w}_{i}, i=1, \ldots, n\right) \\
& =\sum_{t=1}^{T} \sum_{j=1}^{n} G\left(\sum_{i=1}^{n}\left(\mathbf{w}_{i}^{T} \mathbf{x}(t)^{2}\right)\right)+T \log |\operatorname{det} \mathbf{W}| .
\end{aligned}
$$




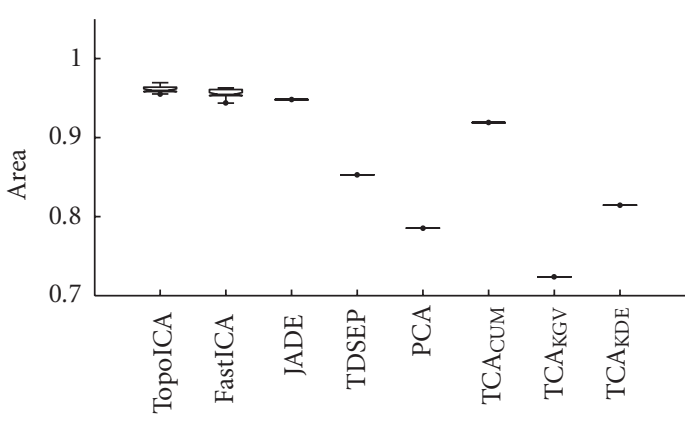

(a)

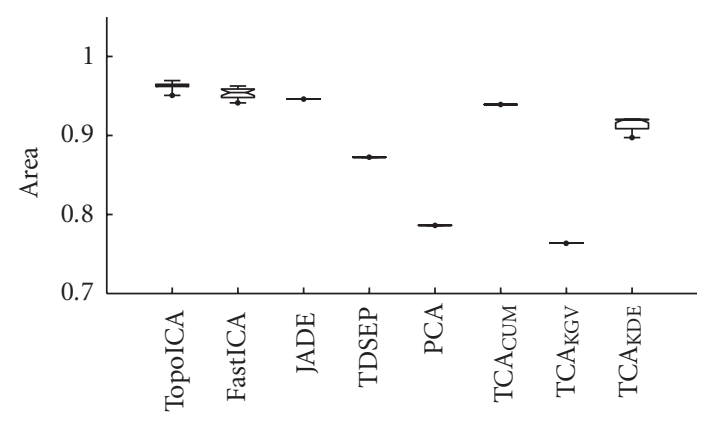

(b)

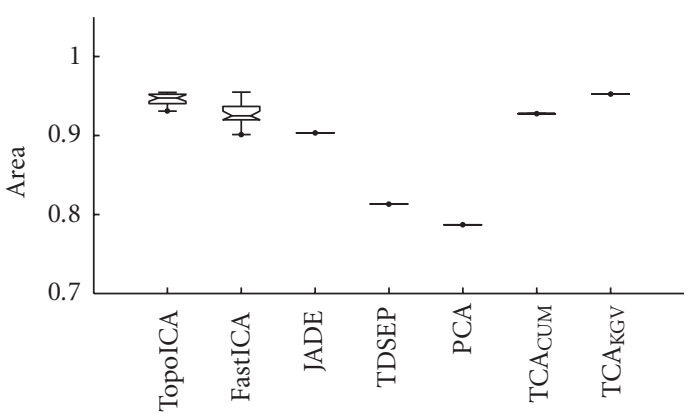

(c)

FIGURE 3: Results of the comparison between tree-dependent ICA, topographic ICA, Jade, FastICA, TDSEP, and PCA on fMRI data. Spatial accuracy of ICA maps is assessed by ROC analysis using correlation map with a chosen threshold of 0.4. The number of chosen independent components (ICs) for all techniques is $N=8$ in (a), $N=9$ in (b), and $N=16$ in (c).

The update rule for the weight vector $\mathbf{w}_{i}$ is derived from a gradient algorithm based on the log likelihood assuming $\log |\operatorname{det} \mathbf{W}|=0$ :

$$
\Delta \mathbf{w}_{i} \propto E\left\{\mathbf{x}\left(\mathbf{w}_{i}^{T} \mathbf{x}\right) r_{i}\right\}
$$

where

$$
r_{i}=\sum_{k=1}^{n} h(i, k) g\left(\sum_{j=1}^{n} h(k, j)\left(\mathbf{w}_{j}^{T} \mathbf{x}\right)^{2}\right)
$$

The function $g$ is the derivative of $G=-\alpha_{1} \sqrt{u}+\beta_{1}$. After every iteration, the vectors $\mathbf{w}_{i}$ in (17) are normalized to unit variance and orthogonalized. This equation represents a modulated learning rule, where the learning term is modulated by the term $r_{i}$.

The classic ICA results from the topographic ICA by setting $h(i, j)=\delta_{i j}$.

\section{RESULTS AND DISCUSSION}

fMRI data were recorded from six subjects (3 female, 3 male, age 20-37) performing a visual task. In five subjects, five slices with 100 images $(\mathrm{TR} / \mathrm{TE}=3000 / 60 \mathrm{msec})$ were acquired with five periods of rest and five photic simulation periods with rest. Simulation and rest periods comprised 10 repetitions each, that is, 30 seconds. Resolution was $3 \times 3 \times 4 \mathrm{~mm}$. The slices were oriented parallel to the calcarine fissure. Photic stimulation was performed using an $8 \mathrm{~Hz}$ alternating checkerboard stimulus with a central fixation point and a dark background with a central fixation point during the control periods [17]. The first scans were discarded for remaining saturation effects. Motion artifacts were compensated by automatic image alignment (AIR, [28]).

The clustering results were evaluated by (1) task-related activation maps, (2) associated time courses, and (3) ROC curves.

\subsection{Estimation of the ICA model}

To decide to what extent spatial ICA of fMRI time series depends on the employed algorithm, we have first to look at the optimal number of principal components selected by PCA and used in the ICA decomposition. ICA is a generalization of PCA. In case no ICA is performed, then the number of independent components equals zero, and this means there is no PCA decomposition performed.

In the following we will give the set parameters. For PCA, no parameters had to be set. For FastICA we choose 


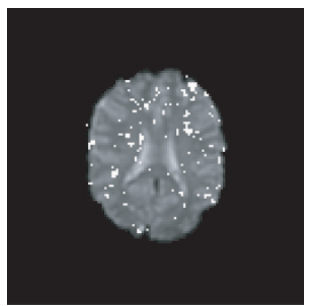

(a)

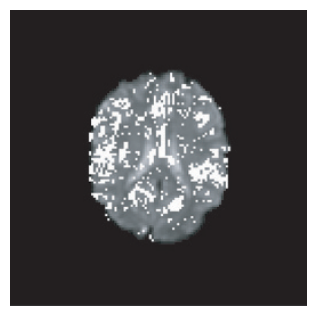

(e)

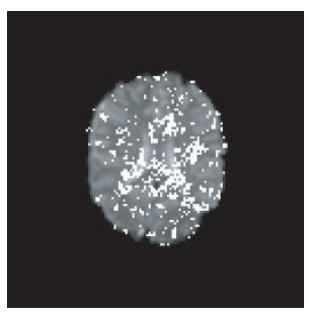

(i)

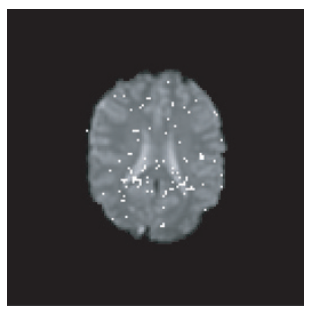

(m)

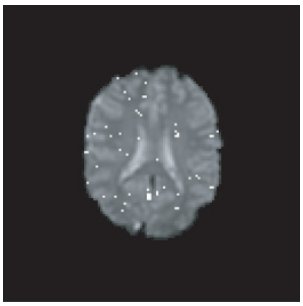

(b)

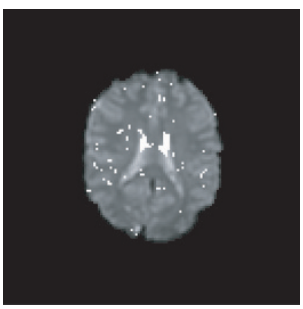

(f)

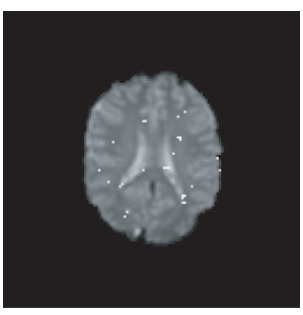

(j)

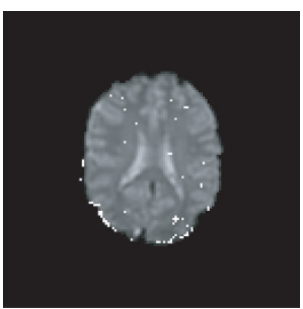

(n)

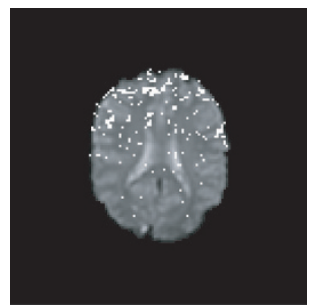

(c)

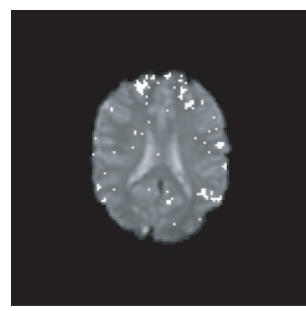

(g)

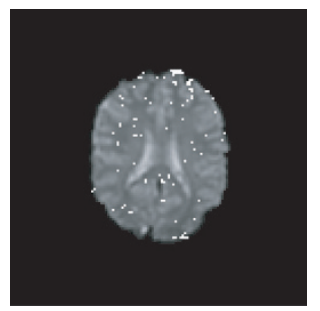

(k)

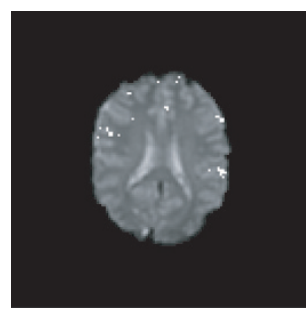

(o)

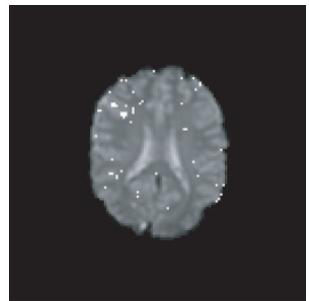

(d)

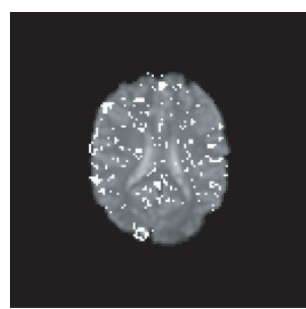

(h)

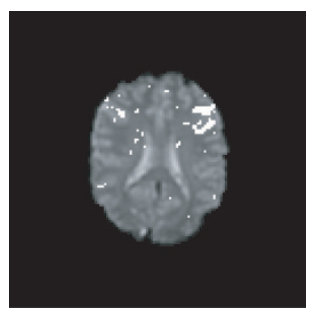

(1)

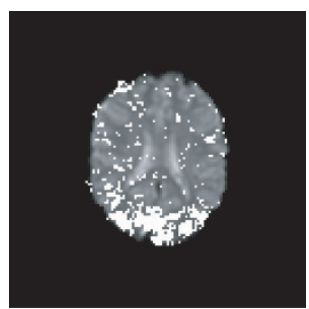

(p)

FIGURE 4: Cluster assignment maps for cluster analysis based on the tree-dependent ICA (CUM) of a visual stimulation fMRI experiment obtained for 16 ICs.

(1) $\epsilon=10^{-6}$, (2) $10^{5}$ as the maximal number of iterations, and (3) the nonlinearity $g(u)=\tanh u$. And last, for topographic ICA we set the following: (1) stop criterium is fullfilled if the synaptic weights difference between two consecutive iterations is less than $10^{-5} \times$ number of ICs, (2) the function $g(u)=u$, and (3) $10^{4}$ is the maximal number of iterations.

It is significant to find a fixed number of ICs that can theoretically predict new observations in same conditions, assuming the basic ICA model actually holds. To do so, we compared the six proposed algorithms for 8,9 , and 16 components in terms of ROC analysis using a correlation map with a chosen threshold of 0.4 . The obtained results are plotted in Figure 3. It can be seen that topographic ICA outperforms all other ICA methods for 8 and 9 ICs. However, for 16 ICs topographic ICA is outperformed by tree-dependent ICA (KGV) using as an approximation of the mutual information the kernel generalized variance.

The clustering results for the two methods, the treedependent (CUM and KGV) and topographic ICA are shown 


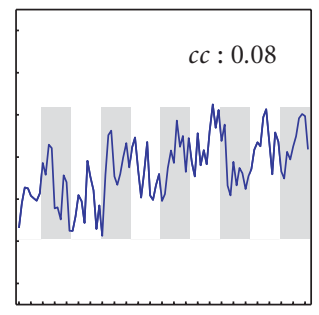

(a)

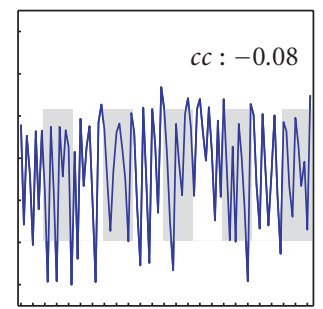

(e)

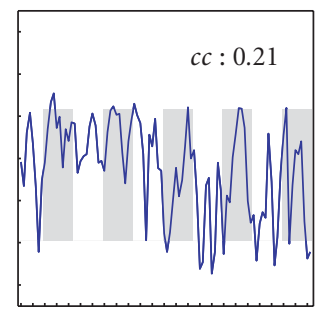

(i)

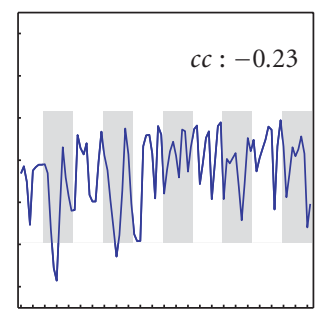

$(\mathrm{m})$

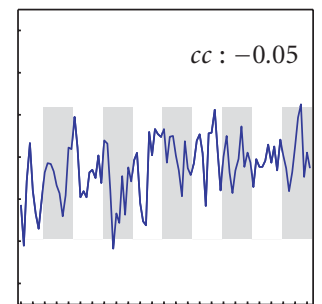

(b)

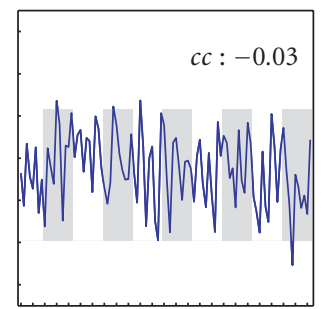

(f)

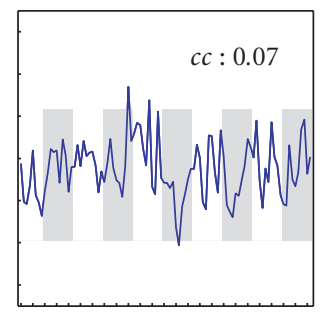

(j)

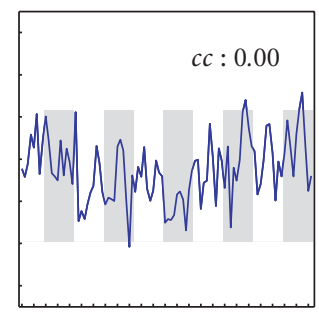

(n)

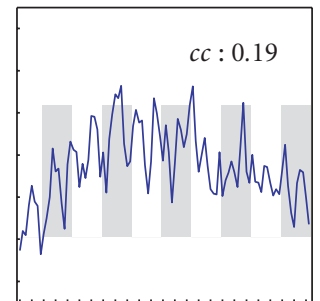

(c)

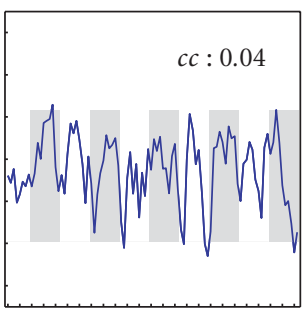

$(\mathrm{g})$

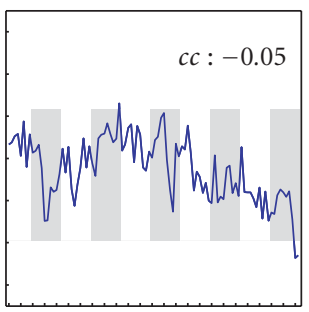

(k)

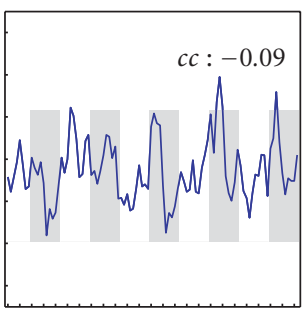

(o)

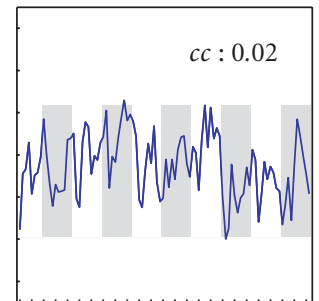

(d)

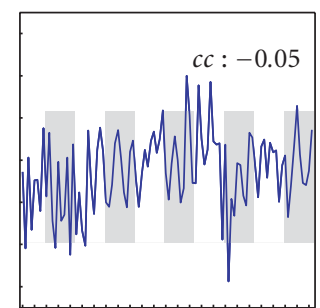

(h)

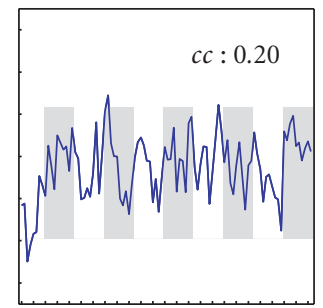

(1)

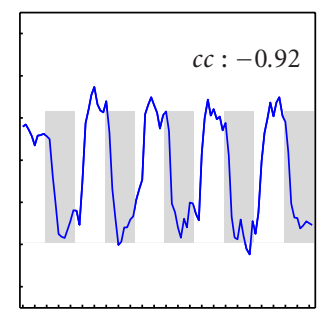

(p)

Figure 5: Associated codebook vectors for the tree-dependent ICA (CUM) as shown in Figure 4. Assignment of the codebook vectors corresponds to the order of the assignment maps shown in Figure 4.

in Figures 4-9. Figures 4, 6, and 8 illustrate the so-called assignment maps where all the pixels belonging to a specific cluster are highlighted. The assignment between a pixel and a specific cluster is given by the minimum distance between the pixel and an IC from the established codebook. On the other hand, each IC shown in Figures 5, 7, and 9 can be viewed as the cluster-specific weighted average of all pixel time courses.

We immediately can see a topographical representation in Figure 9 by looking at the last row (ICs 15 and 16): the two time courses $s$ with the highest absolute-valued correlation are grouped together. Thus, the advantage of the tree-dependent ICA (KGV) becomes immediately evident: it groups together signals according to their dependence content. This effect cannot be observed neither with topographic nor with tree-dependent ICA (CUM).

\subsection{Characterization of task-related effects}

For all subjects, and runs, unique task-related activation maps and associated time courses were obtained by the 


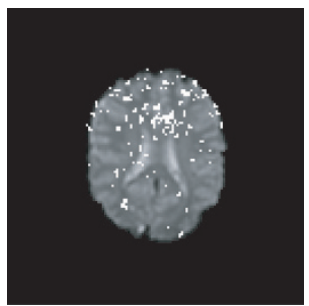

(a)

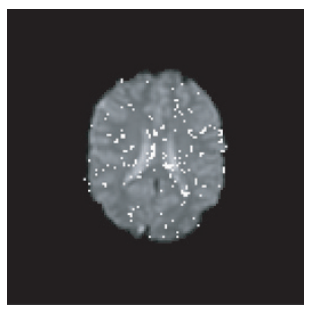

(e)

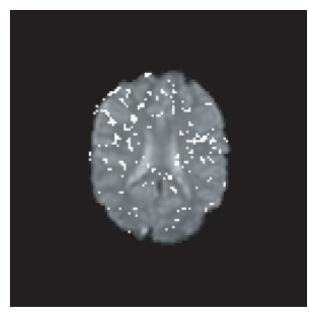

(i)

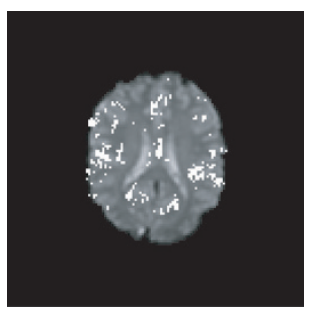

(m)

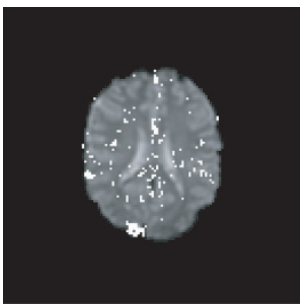

(b)

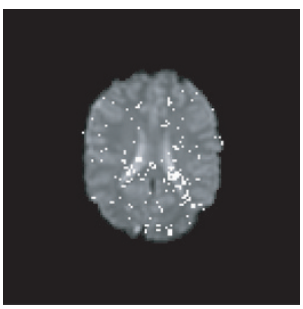

(f)

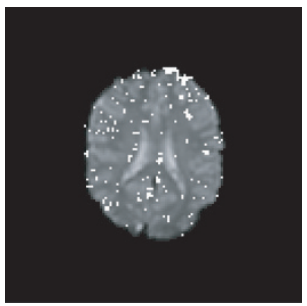

(j)

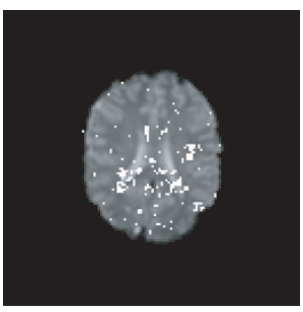

(n)

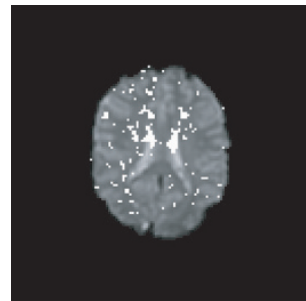

(c)

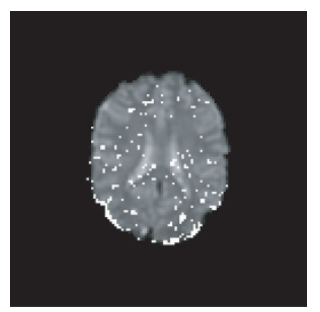

(g)

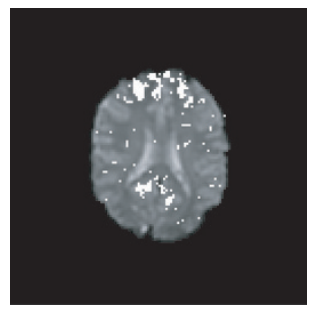

(k)

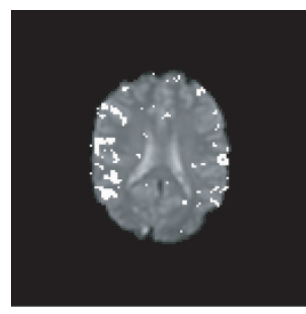

(o)

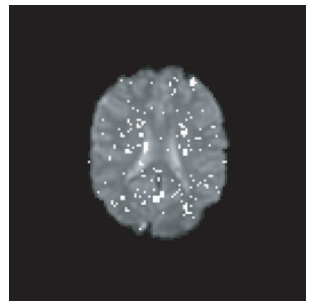

(d)

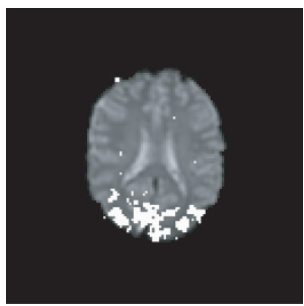

(h)

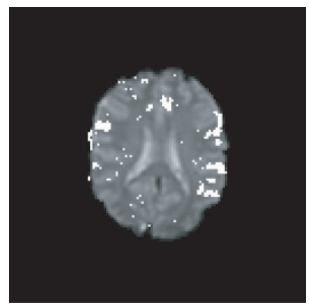

(1)

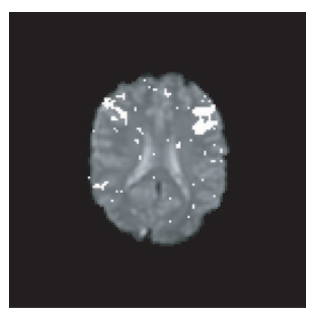

(p)

FIGURE 6: Cluster assignment maps for cluster analysis based on the topographic ICA of a visual stimulation fMRI experiment obtained for 16 ICs.

tree-dependent and topographic ICA techniques. The correlation of the component time course most closely associated with the visual task for these two techniques is shown in Table 1 for $\mathrm{IC}=8,9$, and 16. This time course can serve as an estimate of the stimulus reference function used in the fMRI experiment, as identified by the specific dependent component technique. From Table 1, we see for the treedependent ICA (CUM) a continuous increase for the correlation coefficient while for the topographic ICA this correlation coefficient decreases for IC $=16$ and for tree-dependent $\mathrm{ICA}(\mathrm{KGV})$ it decreases even for $\mathrm{IC}=9$.

\subsection{Exploratory analysis of ancillary findings}

From Figures 4-9, we can also obtain some insight in the type of artifactual components. For the cluster assignment maps in Figure 4, cluster 12 and cluster 16 in Figure 6 may be assigned to a coactivation of the frontal eye fields induced by stimulus onset. No such findings can be reported from Figure 8. There may be some type of physiological relatedness between cluster 12 on one hand, and between cluster 16 showing high correlation with the stimulus function, on the other hand in Figure 4. The same is valid for cluster 16 and cluster 8 in Figure 6. Interestingly, Figure 8 determines two 


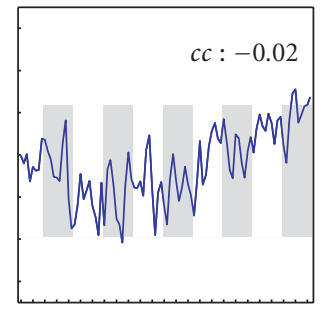

(a)

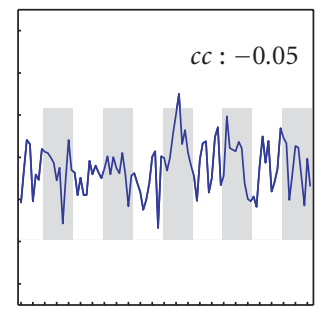

(e)

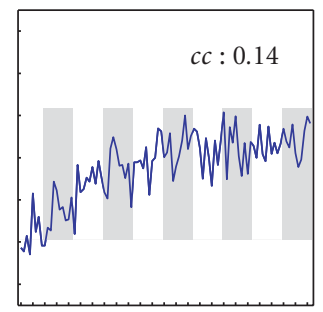

(i)

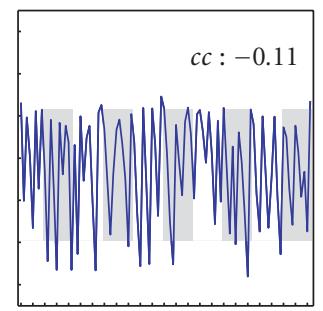

(m)

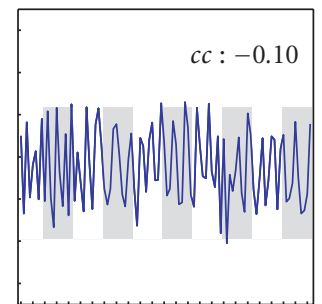

(b)

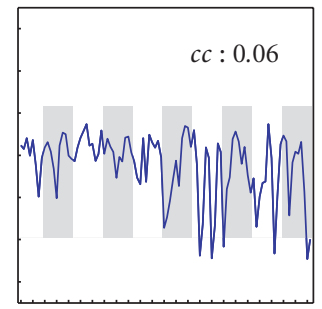

(f)

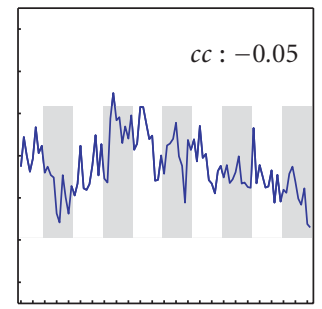

(j)

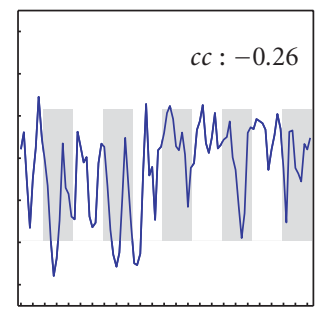

(n)

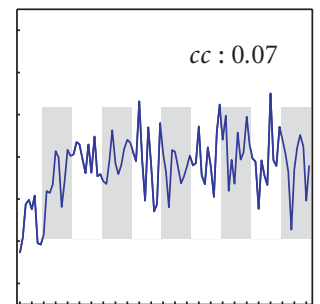

(c)

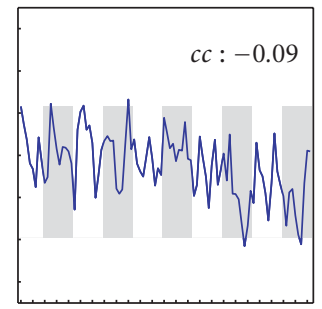

(g)

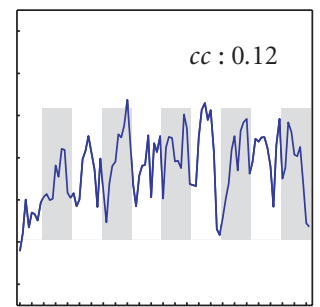

$(\mathrm{k})$

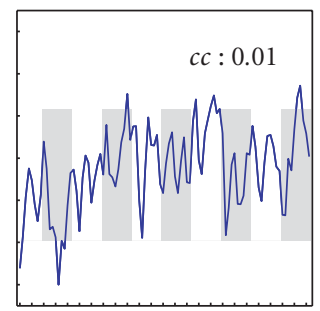

(o)

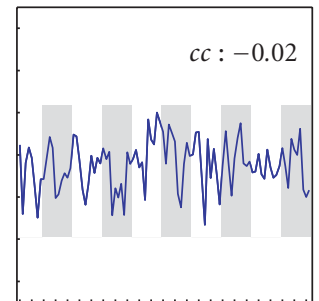

(d)

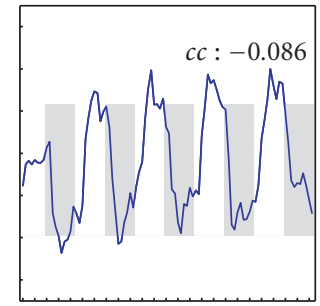

(h)

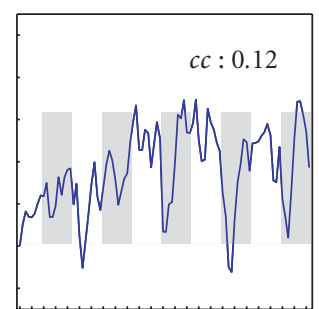

(1)

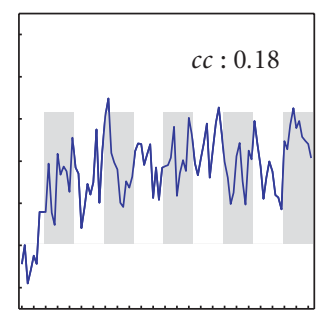

(p)

FIGURE 7: Associated codebook vectors for the topographic ICA as shown in Figure 6. Assignment of the codebook vectors corresponds to the order of the assignment maps shown in Figure 6.

ICs showing a high correlation with the stimulus function. However, this connection is not revealed by the feature space metric and thus is not supported by clustering approaches based on this metric.

An additional benefit from unsupervised clustering techniques represents the ability to identify data highly indicative of artifacts, for example, ventricular pulsation or through plane motion. Cluster 6 in Figure 4 and cluster 3 in Figure 6, for example, show the region of the inner ventricles. It is important to mention that these effects could not have been detected by model-based approaches.

\section{CONCLUSION}

In the present paper, we have experimentally compared four standard ICA algorithms already adopted in the fMRI literature with two new algorithms, the tree-dependent and topographic ICA. The goal of the paper was to determine the robustness and reliability of extracting task-related activation maps and time courses from fMRI data sets. The success of ICA methods is based on the condition that the spatial distribution of brain areas activated by task performance must be spatially independent of the distributions of areas affected 


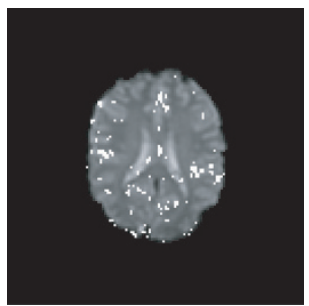

(a)

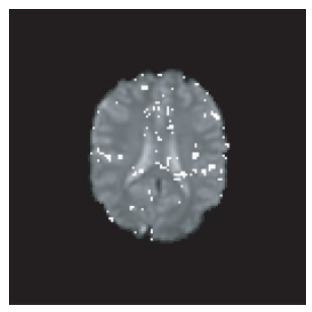

(e)

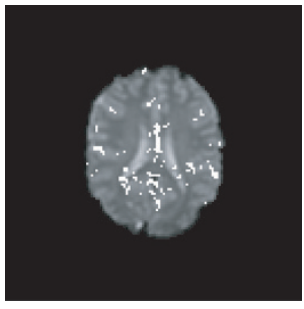

(i)

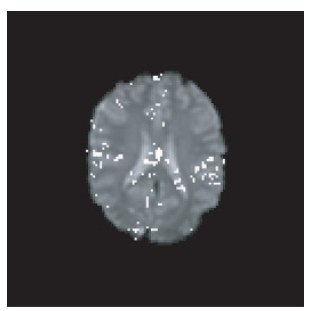

(m)

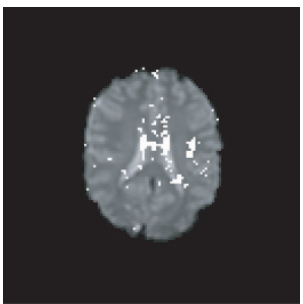

(b)

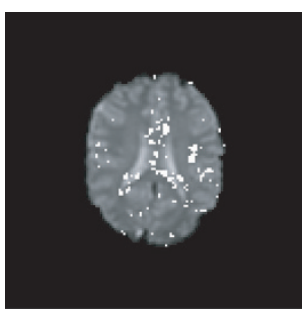

(f)

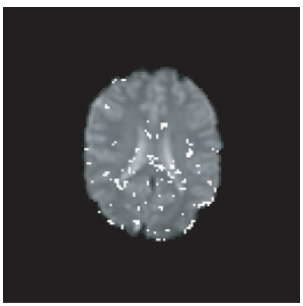

(j)

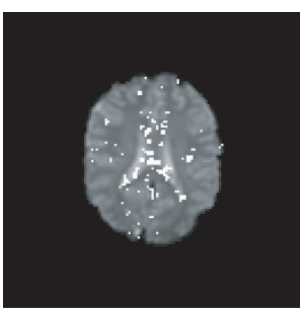

(n)

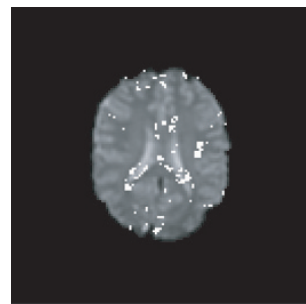

(c)

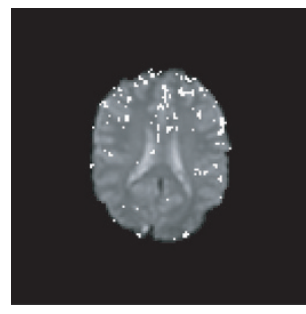

(g)

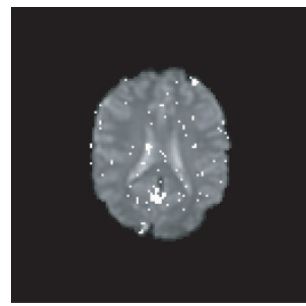

(k)

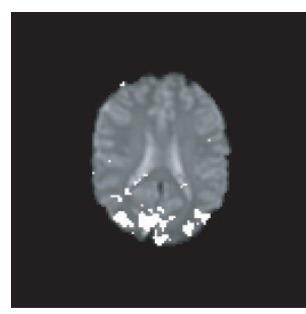

(o)

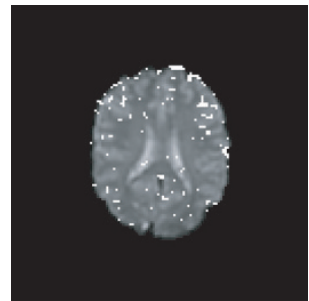

(d)

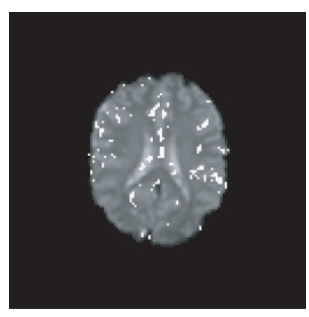

(h)

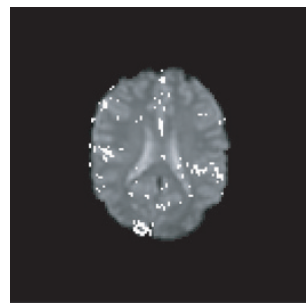

(1)

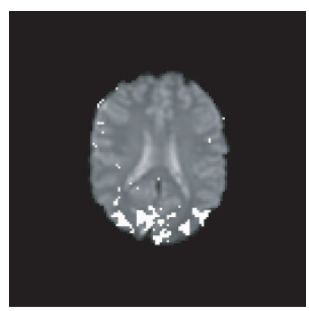

(p)

FIGURE 8: Cluster assignment maps for cluster analysis based on the tree-dependent ICA (KGV) of a visual stimulation fMRI experiment obtained for 16 ICs.

by artifacts. The obtained results proved to reveal extremely well the structure of the data set.

It can be seen that topographic ICA outperforms all other ICA methods for 8 and 9 ICs. However, for 16 ICs topographic ICA is outperformed by tree-dependent ICA (KGV) using as an approximation of the mutual information the kernel generalized variance. All dependent component techniques can be employed to identify interesting ancillary findings that cannot be detected by model-based approaches. The applicability of the new algorithms is demonstrated on experimental data. We conjecture that the method can serve as a multipurpose exploratory data analysis strategy to image time-series analysis and provide good visualization for many fields ranging from biomedical basic research to clinical assessment of patient data. In particular, beyond the application to fMRI data analysis discussed in this paper, the method exhibits a specific potential to serve in applications refering to dynamic contrast-enhanced perfusion MRI for the diagnosis of cerebrovascular disease or magnetic resonance mammography for the analysis of suspicious lesions in patients with breast cancer. In addition, it could yield a visualization of large trees using a hyperbolic space by employing a hyperbolic self-organized map [29]. 


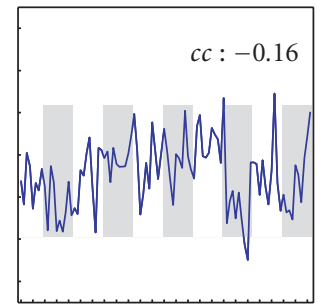

(a)

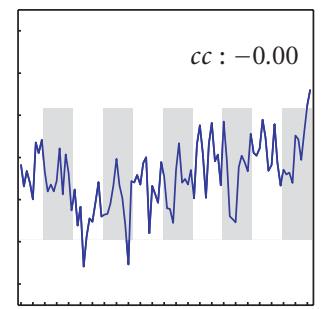

(e)

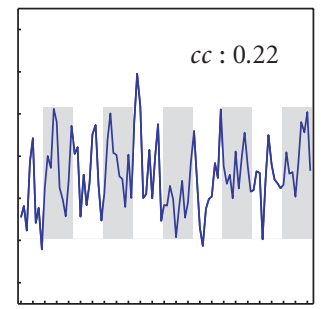

(i)

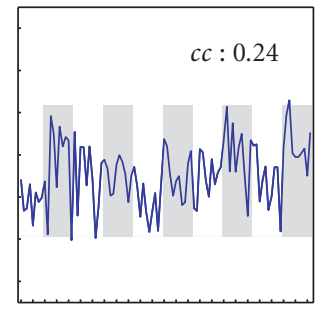

(m)

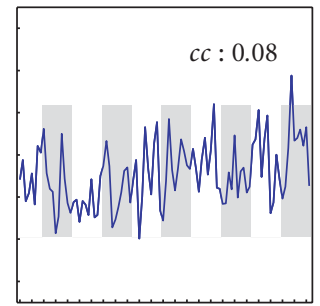

(b)

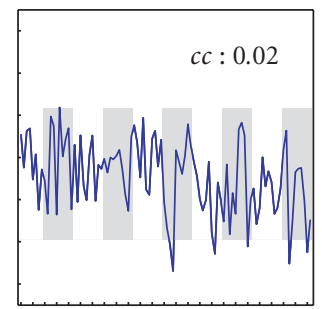

(f)

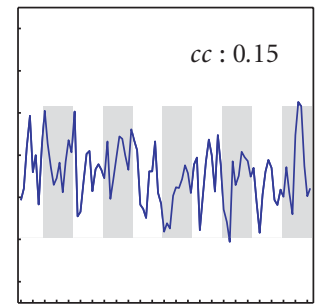

(j)

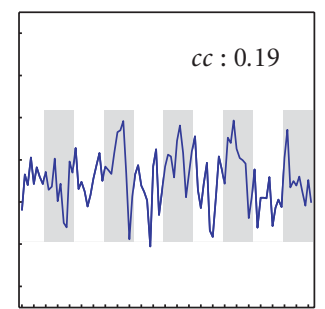

(n)

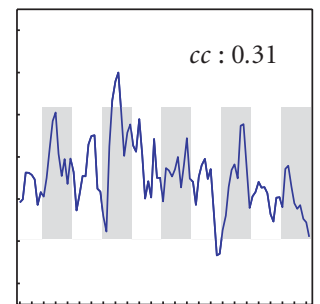

(c)

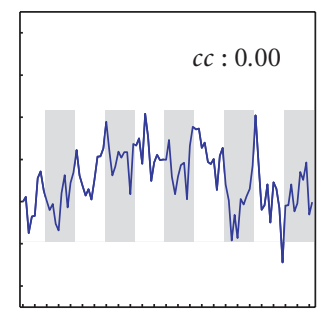

(g)

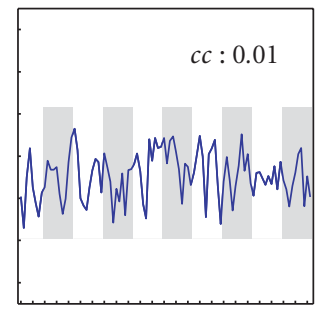

(k)

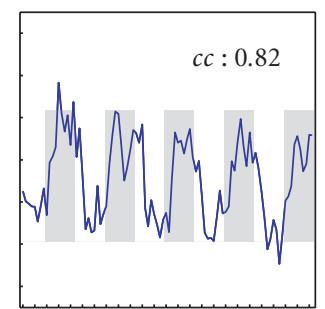

(o)

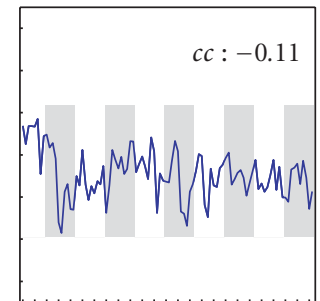

(d)

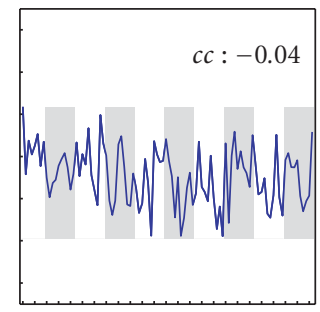

(h)

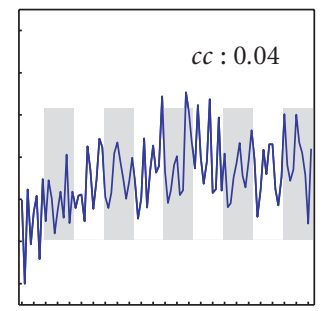

(l)

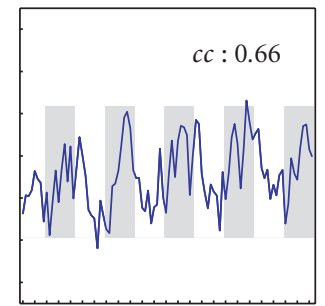

(p)

FIGURE 9: Associated codebook vectors for the tree-dependent ICA (KGV) as shown in Figure 8. Assignment of the codebook vectors corresponds to the order of the assignment maps shown in Figure 8.

TABLE 1: Comparison of the correlations of the component time course most closely associated with the visual task for tree-dependent (tree ICA) and topographic ICA (topo ICA) for IC $=8,9$, and 16 .

\begin{tabular}{lcccc}
\hline No. of ICs & Tree ICA $(\mathrm{KDE})$ & Tree ICA $(\mathrm{KGV})$ & Tree ICA $(\mathrm{CUM})$ & Topo ICA \\
\hline IC $=8$ & 0.78 & 0.74 & 0.78 & 0.85 \\
IC $=9$ & 0.79 & 0.66 & 0.91 & 0.87 \\
IC $=16$ & - & 0.82 & 0.92 & 0.86 \\
\hline
\end{tabular}




\section{ACKNOWLEDGMENTS}

The authors would like to thank Dr. Dorothee Auer from the Max Planck Institute of Psychiatry in Munich, Germany, for providing the fMRI data. We are grateful for the financial support of the Humboldt Foundation.

\section{REFERENCES}

[1] P. A. Bandettini, E. C. Wong, R. S. Hinks, R. S. Tikofsky, and J. S. Hyde, "Time course EPI of human brain function during task activation," Magnetic Resonance in Medicine, vol. 25, no. 2, pp. 390-397, 1992.

[2] J. Frahm, K. D. Merboldt, and W. Hanicke, "Functional MRI of human brain activation at high spatial resolution," Magnetic Resonance in Medicine, vol. 29, no. 1, pp. 139-144, 1993.

[3] K. Kwong, "Functional magnetic-resonance-imaging with echo-planar imaging," Magnetic Resonance Quarterly, vol. 11, no. 1, pp. 1-20, 1995.

[4] K. Kwong, J. Belliveau, D. Chesler, et al., "Dynamic magnetic resonance imaging of human brain activity during primary sensor stimulation," Proceedings of the National Academy of Science, vol. 89, no. 12, pp. 5675-5679, 1992.

[5] S. Ogawa, D. Tank, R. Menon, et al., "Intrinsic signal changes accompanying sensory stimulation: functional brain mapping with magnetic resonance imaging," Proceedings of the National Academy of Science, vol. 89, no. 13, pp. 5951-5955, 1992.

[6] J. Boxerman, P. A. Bandettini, K. Kwong, et al., "The intravascular contribution to FMRI signal change: Monte Carlo modeling and diffusion-weighted studies in vivo," Magnetic Resonance in Medicine, vol. 34, no. 1, pp. 4-10, 1995.

[7] S. Ogawa, T. Lee, and B. Barrere, "The sensitivity of magnetic resonance image signals of a rat brain to changes in the cerebral venous blood oxygenation activation," Magnetic Resonance in Medicine, vol. 29, no. 2, pp. 205-210, 1993.

[8] J. J. Sychra, P. A. Bandettini, N. Bhattacharya, and Q. Lin, "Synthetic images by subspace transforms I. Principal components images and related filters," Medical Physics, vol. 21, no. 2, pp. 193-201, 1994.

[9] W. Backfrieder, R. Baumgartner, M. Sámal, E. Moser, and H. Bergmann, "Quantification of intensity variations in functional MR images using rotated principal components," Physics in Medicine and Biology, vol. 41, no. 8, pp. 1425-1438, 1996.

[10] M. J. McKeown, T.-P. Jung, S. Makeig, et al., "Spatially independent activity patterns in functional MRI data during the stroop color-naming task," Proceedings of the National Academy of Sciences, vol. 95, no. 3, pp. 803-810, 1998.

[11] M. J. McKeown, S. Makeig, G. G. Brown, et al., "Analysis of fMRI data by blind separation into independent spatial components," Human Brain Mapping, vol. 6, no. 3, pp. 160-188, 1998.

[12] F. Esposito, E. Formisano, E. Seifritz, et al., "Spatial independent component analysis of functional MRI time-series: To what extent do results depend on the algorithm used?" $\mathrm{Hu}$ man Brain Mapping, vol. 16, no. 3, pp. 146-157, 2002.

[13] K. Arfanakis, D. Cordes, V. M. Haughton, C. H. Moritz, M. A. Quigley, and M. E. Meyerand, "Combining independent component analysis and correlation analysis to probe interregional connectivity in fMRI task activation datasets," Magnetic Resonance Imaging, vol. 18, no. 8, pp. 921-930, 2000.

[14] G. Scarth, M. McIntyre, B. Wowk, and R. Somorjai, "Detection of novelty in functional images using fuzzy clustering," in Proc. 3rd Scientific Meeting of the International Society for Magnetic Resonance in Medicine, vol. 95, pp. 238-242, Nice, France, August 1995.
[15] K.-H. Chuang, M.-J. Chiu, C.-C. Lin, and J.-H. Chen, "Model-free functional MRI analysis using Kohonen clustering neural network and fuzzy C-means," IEEE Trans. Med. Imag., vol. 18, no. 12, pp. 1117-1128, 1999.

[16] R. Baumgartner, L. Ryner, W. Richter, R. Summers, M. Jarmasz, and R. Somorjai, "Comparison of two exploratory data analysis methods for fMRI: fuzzy clustering vs. principal component analysis," Magnetic Resonance Imaging, vol. 18, no. 1, pp. 89-94, 2000.

[17] A. Wismüller, O. Lange, D. R. Dersch, et al., "Cluster analysis of biomedical image time-series," International Journal of Computer Vision, vol. 46, no. 2, pp. 103-128, 2002.

[18] H. Fischer and J. Hennig, "Clustering of functional MR data," in Proc. 4th Annual Meeting of the International Society for Magnetic Resonance in Medicine (ISMRM '96), pp. 1179-1183, New York, NY, USA, April 1996.

[19] S. C. Ngan and X. Hu, "Analysis of functional magnetic resonance imaging data using self-organizing mapping with spatial connectivity," Magnetic Resonance in Medicine, vol. 41, no. 5, pp. 939-946, 1999.

[20] A. Hyvärinen, P. Hoyer, and M. Inki, "Topographic independent component analysis," Neural Computation, vol. 13, no. 7, pp. 1527-1558, 2001.

[21] F. R. Bach and M. I. Jordan, "Beyond independent components: trees and clusters," Journal of Machine Learning Research, vol. 4, pp. 1205-1233, December 2003.

[22] J.-F. Cardoso, "Multidimensional independent component analysis," in Proc. IEEE International Conference on Acoustics, Speech, and Signal Processing (ICASSP '98), vol. 4, pp. 19411944, Seattle, Wash, USA, May 1998.

[23] A. Hyvärinen and E. Oja, "Independent component analysis: algorithms and applications," Neural Networks, vol. 13, no. 45, pp. 411-430, 2000.

[24] C. R. Chow and C. N. Liu, "Approximating discrete probability distributions with dependence trees," IEEE Trans. Inform. Theory, vol. 14, no. 3, pp. 462-467, 1968.

[25] A. Hyvärinen and P. Hoyer, "Emergence of phase- and shiftinvariant features by decomposition of natural images into independent feature subspaces," Neural Computation, vol. 12, no. 7, pp. 1705-1720, 2000.

[26] T. Kohonen, "Emergence of invariant-feature detectors in the adaptive-subspace self-organizing map," Biological Cybernetics, vol. 75, no. 4, pp. 281-291, 1996.

[27] A. Meyer-Bäse, Pattern Recognition for Medical Imaging, Academic Press, Boston, Mass, USA, 2003.

[28] R. P. Woods, S. R. Cherry, and J. C. Mazziotta, "Rapid automated algorithm for aligning and reslicing PET images," Journal of Computer Assisted Tomography, vol. 16, no. 4, pp. 620633, 1992.

[29] H. Ritter, "Self-organizing maps in non-euclidean spaces," in Kohonen Maps, pp. 97-108, Springer, Berlin, Germany, 1999.

Anke Meyer-Bäse is with the Department of Electrical and Computer Engineering at the Florida State University. Her research areas include theory and application of neural networks, medical image processing, pattern recognition, and parallel processing. She was awarded the Lise-MeitnerPrize in 1997. She published over 100 papers in several areas including intelligent systems, medical image processing, speech recognition, and neural networks. She is author of the book Pattern Recognition in Medical Imaging which appeared in Elsevier/Academic Press in 2003. 
Monica K. Hurdal is an Assistant Professor of Biomedical Mathematics at Florida State University in Tallahassee, Florida. She was awarded her Ph.D. degree in 1999 from Queensland University of Technology, Australia, in applied mathematics. Subsequently, Dr. Hurdal was a Postdoctoral Research Associate for two years at Florida State University (FSU) in mathematics and also computer science, working on confor-

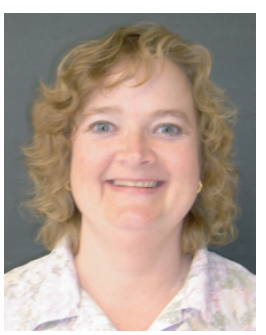
mal flat mapping of the human brain. She continued her research at Johns Hopkins University in the Center for Imaging Science as a Research Scientist, followed by her current position in 2001 in Biomedical Mathematics at FSU. Her research interests include applying topology, geometry, and conformal methods to the analysis and modeling of neuroscientific data from the human brain. She is investigating topology issues associated with constructing cortical surfaces from MRI data, computing conformal maps of the brain, and applying topological and conformal invariants to characterize disease in MRI studies.

Oliver Lange studied information technologies engineering at the TU in Munich. After finishing his diploma in 1999, he was a Ph.D. student in biomedical engineering at the Institute of Clinical Radiology at the University of Munich. When he finished his Ph.D. in 2003, Oliver Lange was a Consultant for the Department of Engineering at Florida State University. Since July 2004, he has been working as a Research Engineer in the field of biomedical signal processing.

Helge Ritter studied physics and mathematics at the Universities of Bayreuth, Heidelberg, and Munich. After a Ph.D. degree in physics at the Technical University of Munich in 1988, he visited the Laboratory of Computer Science at Helsinki University of Technology and the Beckman Institute for Advanced Science and Technology at the University of Illinois at UrbanaChampaign. Since 1990 he has been the

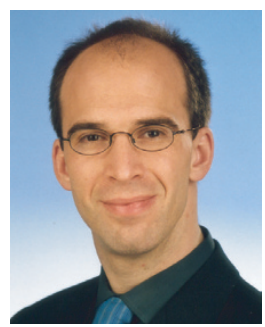
Head of the Neuroinformatics Group at the Faculty of Technology, Bielefeld University. His main interests are principles of neural computation and their application to build intelligent systems. In 1999, Helge Ritter was awarded the SEL Alcatel Research Prize and in 2001 the Leibniz Prize of the German Research Foundation DFG. 